\title{
ESTUDIO DE ALGUNAS PROPIEDADES FÍSICAS Y FISIOLÓGICAS PRECOSECHA DE LA PERA VARIEDAD TRIUNFO DE VIENA ${ }^{1}$
}

\author{
ALFONSO PARRA-CORONADO², JOSÉ EUGENIO HERNÁNDEZ HERNÁNDEZ³, \\ JESÚS HERNÁN CAMACHO-TAMAYO ${ }^{4}$
}

\begin{abstract}
RESUMEN - El objetivo del presente trabajo fue establecer algunas propiedades físicas y fisiológicas de desarrollo de la pera (Pyrus communis L.) variedad Triunfo de Viena, producida bajo las condiciones ecuatoriales del clima frío, en los Andes Colombianos. El crecimiento y propiedades de la fruta fueron evaluados en intervalos de 30 días, desde la floración. Las propiedades estudiadas fueron el peso, tamaño de la fruta, firmeza de la pulpa, concentración de sólidos solubles, pH, acidez titulable (\% ácido málico) e intensidad respiratoria. La concentración de sólidos solubles y el pH no son propiedades sensibles para determinar el momento apropiado de la cosecha, al presentar valores constantes y de baja dispersión. El fruto presenta forma esférica y su área superficial es función directa del peso de la fruta. La firmeza de la pulpa, la acidez titulable (AT) y la intensidad respiratoria, decrecen en la medida que se desarrolla la pera, presentando valores en el momento de la cosecha de 67,82 N para la firmeza, acidez titulable de 0,24\% e intensidad respiratoria de 29,60 $\mathrm{mg} \mathrm{CO}_{2} \mathrm{~kg}^{-1} \mathrm{~h}^{-1}$. Los sólidos solubles (SS) y la relación SS/AT se incrementan en la medida que el fruto va madurando. En el momento de la cosecha los frutos presentan un valor de SS de 12,0Brix, una relación SS/AT de 49,36 y un pH de 3,94. Términos para indexación: Índices de cosecha, sólidos solubles, acidez titulable, firmeza, respiración, Pyrus communis L.
\end{abstract}

\section{PREHARVEST STUDY OF SOME PHYSICAL AND PHYSIOLOGICAL PROPERTIES OF PEAR cV. TRIUNFO DE VIENA}

ABSTRACT - The objective of the present work was to determine some physical and physiological properties of pear development cv. Triunfo de Viena, produced under the equatorial cold at the Colombian Andes. The fruit growth was evaluated at 30 days intervals starting at its full bloom. The studied properties were fruit weight, size, flesh firmness, soluble solids concentration, $\mathrm{pH}$, titratable acids (\% malic acid) and respiratory rate. The soluble solids concentration and $\mathrm{pH}$ are not sensible properties to determine the appropriate harvest time, presenting constant values and low dispersion. The fruit presents spherical form and its superficial area is a direct function of the fruit weight. The flesh firmness, titratable acids (TA) and respiratory rate, decrease when the pear grows, presenting harvest values of 67,82 $\mathrm{N}$ for flesh firmness, titratable acidity of $0,24 \%$ and respiratory rate of $29,60 \mathrm{mg} \mathrm{CO}_{2} \mathrm{~kg}^{-1} \mathrm{~h}^{-1}$. The soluble solids concentration (SS), and the relation SS/TA increase at the fruit ripening. At harvest time the fruits present SS about 12,0 $0^{\circ}$ Brix, relation SS/TA of 49,36 and $\mathrm{pH} 3,94$.

Index terms: harvest index, soluble solids, titratable acids, firmness, respiration, Pyrus communis L.

\section{INTRODUCCIÓN}

En zonas subtropicales o de clima templado, la pera es una de las frutas de mayor importancia económica, junto con la manzana y el durazno. El 2004 presentó una producción mundial de 17,9 millones de toneladas métricas, siendo sus principales productores en el contexto mundial China, Estados Unidos, Italia, España y Argentina (FAO, 2005). En Colombia, este cultivo ha tomado importancia durante los últimos años, debido al continuo incremento en el consumo per cápita de frutas, así como la adaptación de esta especie en diferentes regiones de los Andes, a una altitud entre los 2300 y $2600 \mathrm{~m}$, con temperatura media de $15^{\circ} \mathrm{C}$.

La pera, al igual que otras especies caducifolias, presenta estados definidos de crecimiento entre la floración y la cosecha, como son la división celular, diferenciación entre tejidos, aumento del tamaño y maduración. Una vez se alcanza la madurez, los agricultores realizan la cosecha mediante indicadores como coloración de la epidermis, tamaño y peso de la fruta, parámetros que no son adecuados para definir el momento propicio de la cosecha, debido a la irregularidad de los frutos (Kabas \& Ozmerzi, 2005), especialmente para la venta de este producto en mercados internacionales.

Para la determinación de la madurez de las frutas en general, es necesario considerar otros parámetros como firmeza de la pulpa, acidez titulable, contenido de sólidos solubles, así como la intensidad respiratoria (Lombardi et al., 2000; Parra \& Hernández, 1997), donde la relación entre los sólidos solubles y la acidez titulable, es un parámetro apropiado para la determinación de la calidad de la pera (Kappel et al., 1995). Estas propiedades son influenciadas por las condiciones climáticas, especialmente por la luminosidad y la temperatura (Calvo, 2004) e inciden directamente en la formación del fruto y en parámetros como la firmeza, color y en la concentración de sólidos solubles (Kappel y Neilsen, 1994), así como en la conservación durante las operaciones de poscosecha (Park et al., 2002).

En Colombia la floración de los árboles de pera se da entre septiembre y noviembre y la cosecha se realiza entre los meses de marzo y junio del siguiente año. Dada la situación geográfica de Colombia, no se cuenta con periodos de alta luminosidad, ni el cultivo se ve sometido a cambios de temperatura a lo largo del año, marcado por la estaciones, como sucede en los principales países productores de pera. Por esto, el presente trabajo tuvo como objetivo establecer algunas características físicas y fisiológicas de crecimiento, desde la floración hasta la cosecha, de la pera variedad Triunfo de Viena, cultivada bajo las condiciones ecuatoriales del clima frío.

\section{MATERIALYMÉTODOS}

El estudio fue realizado con frutas de pera obtenidas en el Municipio de Nuevo Colón, ubicado en el Departamento de Boyacá (Colombia), en las coordenadas geográficas $5^{\circ} 21$ ' 11 '” de latitud norte, $73^{\circ} 27^{\prime} 24^{\prime \prime}$ de longitud oeste y una altitud de $2464 \mathrm{~m}$, el cual presenta una temperatura media de $15^{\circ} \mathrm{C}$ y una precipitación media anual de 895,4 $\mathrm{mm}$. Inicialmente se tomaron muestras de seis sitios donde se realizaron la determinación de las propiedades físicas básicas (dimensiones y peso) y de la intensidad respiratoria; con esta información fue posible determinar mediante análisis de distribución F y distribución t de Student,

\footnotetext{
${ }^{1}$ (Trabalho 121/2005). Recebido: 16/08/2005. Aceito para publicação: 08/03/2006.

${ }^{2}$ Ing. Agrícola, M. Sc., Universidad Nacional de Colombia, Profesor Titular, Programa de Ingeniería Agrícola, Ciudad Universitaria, Bogotá, Colombia. aparrac@unal.edu.co. Teléfono 57-1-3165430.

${ }^{3}$ Ing. Agrícola, M. Sc., Universidad Nacional de Colombia, Profesor Asociado, Programa de Ingeniería Agrícola, Ciudad Universitaria, Bogotá, Colombia.jehernandezh@unal.edu.co.

${ }^{4}$ Ing. Agrícola, M. Sc., Universidad Nacional de Colombia, Profesor Asistente, Programa de Ingeniería Agrícola, Ciudad Universitaria, Bogotá, Colombia.jhcamachot@unal.edu.co.
} 


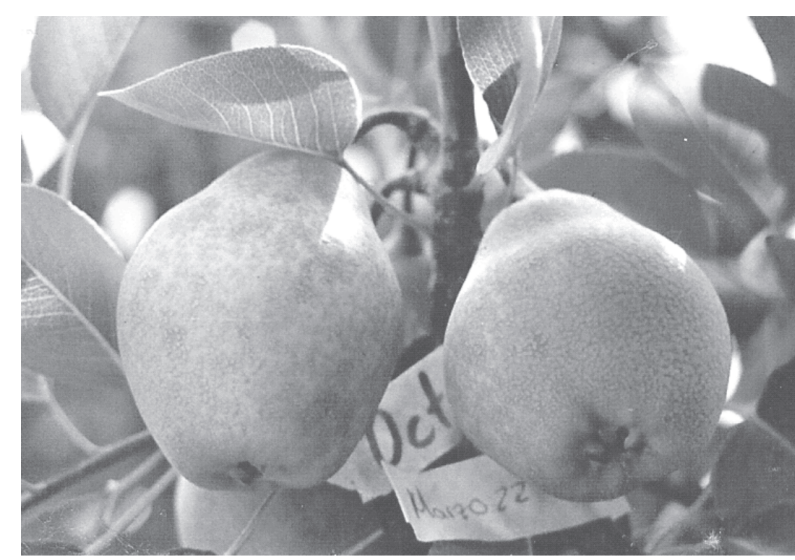

FIGURA 1 - Pera variedad Triunfo de Viena.

la igualdad entre las muestras tomadas de las dos fincas y entre los lotes de una misma finca, lo cual permitió realizar el muestreo de manera aleatoria en las zonas de muestreo.

Luego se realizó en campo la marcación de racimos en plena floración, para 25 árboles por zona, de donde se tomaron 25 frutos cada 30 días durante cinco meses. Las propiedades físicas y fisiológicas se determinaron en el Laboratorio de Poscosecha de Frutas y Hortalizas del Departamento de Ingeniería Civil y Agrícola de la Universidad Nacional de Colombia, Bogotá

Para las propiedades físicas se utilizó la metodología propuesta por Mohsenin (1986), determinando forma (esfericidad y redondez), dimensiones de la fruta (Figura 1), considerando la altura (A) y el diámetro en dos posiciones perpendiculares (B y $\mathrm{C}$ ), peso unitario, densidad aparente, densidad real, porosidad y área superficial real. Los parámetros firmeza de la pulpa, sólidos solubles (SS), pH, acidez titulable (AT), relación de madurez (SS/AT) e intensidad respiratoria (IR), se determinaron utilizando la metodología propuesta por Parra \& Hernández (1997), tomando 25 muestras, cada 30 días, por un periodo de 150 días.

Los resultados fueron analizados mediante estadísticos descriptivos, considerando el coeficiente de variación (CV), como factor de dispersión. También se realizó el análisis de correlación entre diferentes parámetros, para identificar la relación entre estos y establecer cuales serían los mejores indicadores para la cosecha de la pera.

\section{RESULTADOSYDISCUSIÓN}

Características precosecha. Los frutos de pera, al igual que todos los frutos, presentan incrementos continuos en su tamaño y peso a través del tiempo (Figura 2). Cuando los frutos tenían un mes de edad, presentaron una dimensión media de $37,2 \mathrm{~mm}$ y un peso medio de 28,5 g, con CV de 6,7\% y 15,8\% respectivamente, correspondiente a la división celular, para luego presentar la diferenciación de los tejidos durante el segundo mes, donde presentó un tamaño medio de 51,0 mm y peso medio de 70,2 g. Para los siguientes dos meses se observa una ganancia continua de peso y tamaño, tiempo en el cual se da inicio a la maduración propia del fruto. Durante el quinto mes es donde se presenta la mayor ganancia de peso, correspondiente a un 41,2\% del peso final, alcanzando una media final de 312,3 $\pm 58,4 \mathrm{~g}$. Los CV obtenidos para el tamaño oscilan entre $5,0 \%$ y 7,7\% y se van incrementando con la edad de los frutos, lo cual indica que estas propiedades físicas son homogéneas entre los diferentes frutos a medida que estos se desarrollan en la planta, comportamiento también observado por Marsal et al. (2000) y Mwaniki et al. (2005) para las variedades Barlett y Le France. El peso presenta CV relativamente altos, los cuales se van acentuando a medida que se desarrolla el fruto. Este comportamiento de tamaño y peso es similar al presentado por la pera variedad Shinsseiki (Lombardi et al., 2000) y dejan ver la importancia de realizar la cosecha en el momento apropiado, pues de realizarse una cosecha temprana los rendimiento serían menores, además de dificultar el manejo poscosecha del mismo. El diámetro medio final para la variedad Triunfo de Viena fue de 81,98 \pm
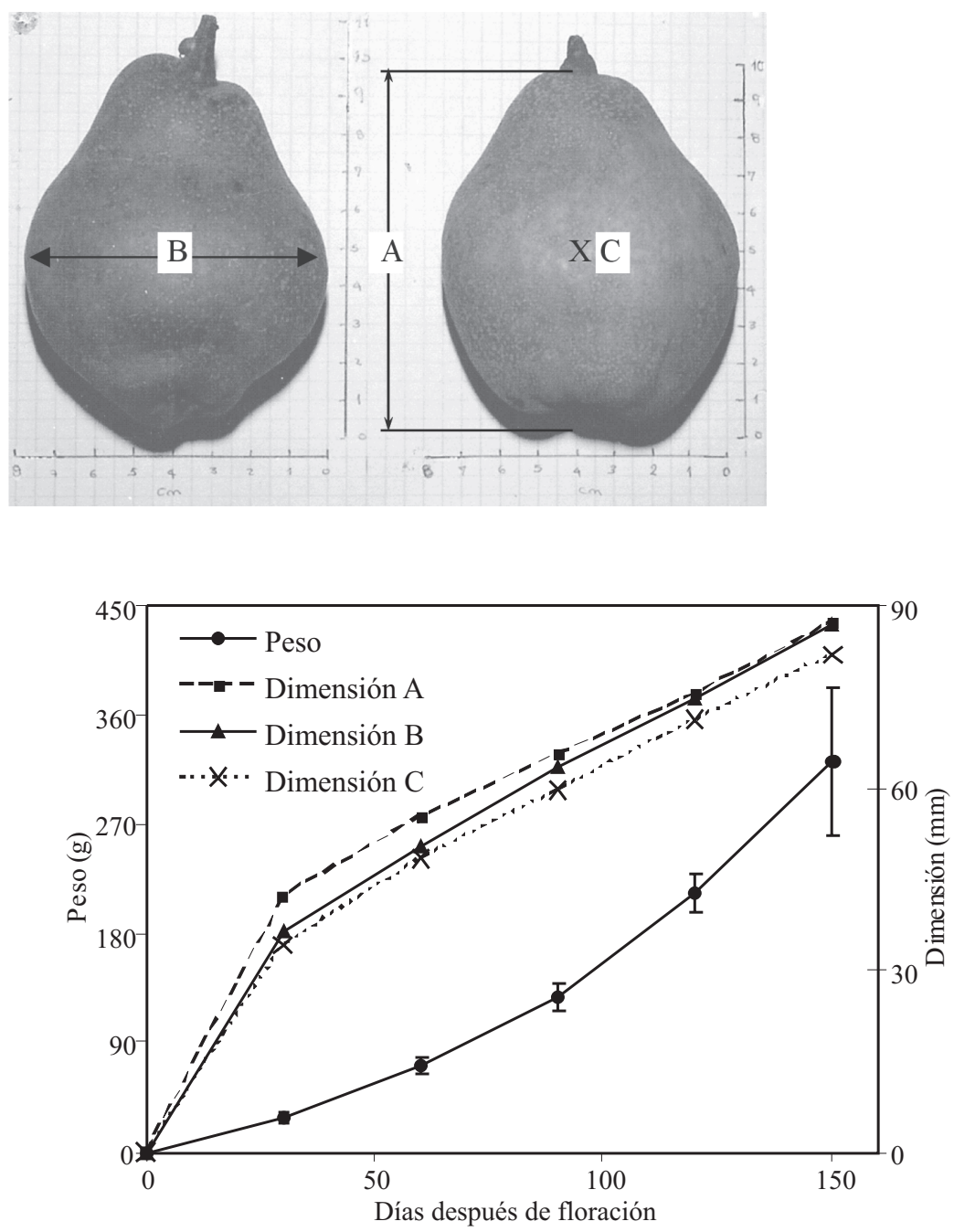

FIGURA 2 - Medias de las dimensiones durante el crecimiento y evolución del peso desde floración y hasta la cosecha. Las barras presentan la media \pm sd.

5,86 mm y de acuerdo con Kappel et al. (1995) estaría por encima del deseable por los consumidores de pera, el cual fue determinado por estos autores entre 60 y $75 \mathrm{~mm}$, indicando que para la cosecha de esta variedad, con fines de mercados internacionales, sería deseable realizar su cosecha antes de los 150 días. Para el caso de la pera este es un índice sensible y homogéneo, con CV de 3,3\% en los primeros estados de crecimiento y que aumenta hasta alcanzar un valor de $8,5 \%$ en el momento de la cosecha, comportamiento también reportado por Garriz et al (1998) para la pera variedad Barllet.

La firmeza presenta valores altos al primer mes (121,12 N), para decrecer a medida que el fruto se desarrolla, hasta alcanzar un valor medio de 67,82 \pm 6,88 $\mathrm{N}$ en el momento de la cosecha, valores que son similares a los reportados por Seibert et al. (2000) para la variedad Packham's Triumph (72,0 N). Este comportamiento se debe a que las sustancias cementantes que le proporcionan la turgencia al fruto (protopectinas y pectinas) se transforman en ácidos pécticos solubles en agua y otras sustancias, produciendo el característico ablandamiento de la fruta madura. La Figura 3 presenta el comportamiento de la firmeza de los frutos de pera durante el periodo precosecha.

El contenido del ácido málico (acidez titulable), decrece en la medida que el fruto se desarrolla (Figura 3), debido a que los ácidos orgánicos presentes se van transformando en otras sustancias (azúcares) en los procesos de respiración. Los altos valores del CV, los cuales oscilan entre 10,3\% y 20,0\% para las diferentes edades, indican que este parámetro es heterogéneo en frutos de la misma edad. El comportamiento de este parámetro concuerda con los resultados reportados por Lombardi et al. (2000).

En general para el periodo precosecha, los SS y el pH presentan una tendencia relativamente uniforme y de baja dispersión (Figura 4), lo 


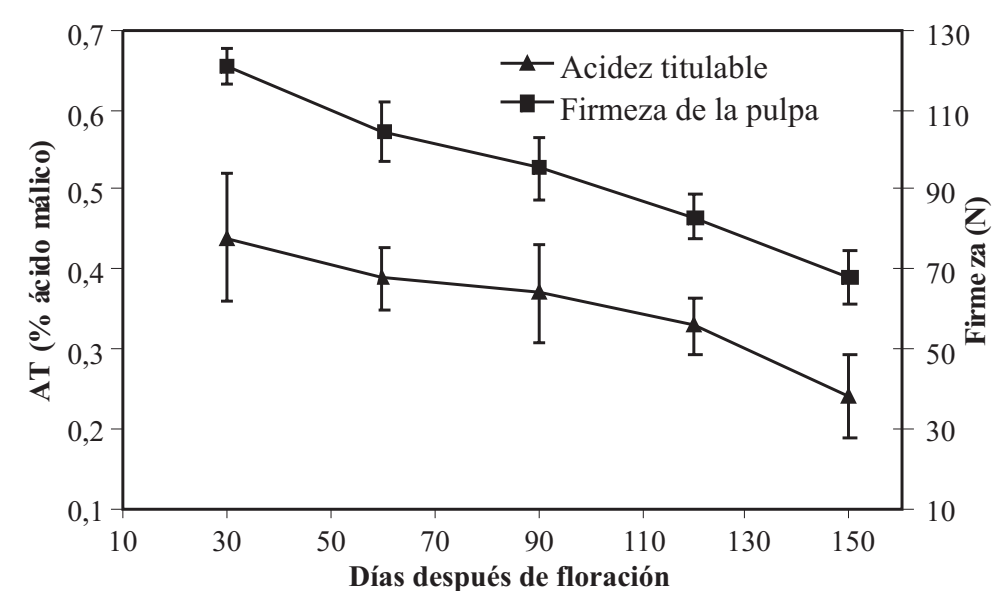

FIGURA 3 - Comportamiento de la acidez titulable y de firmeza de la pulpa de la pera variedad Triunfo de Viena. Las barras presentan la media \pm sd.

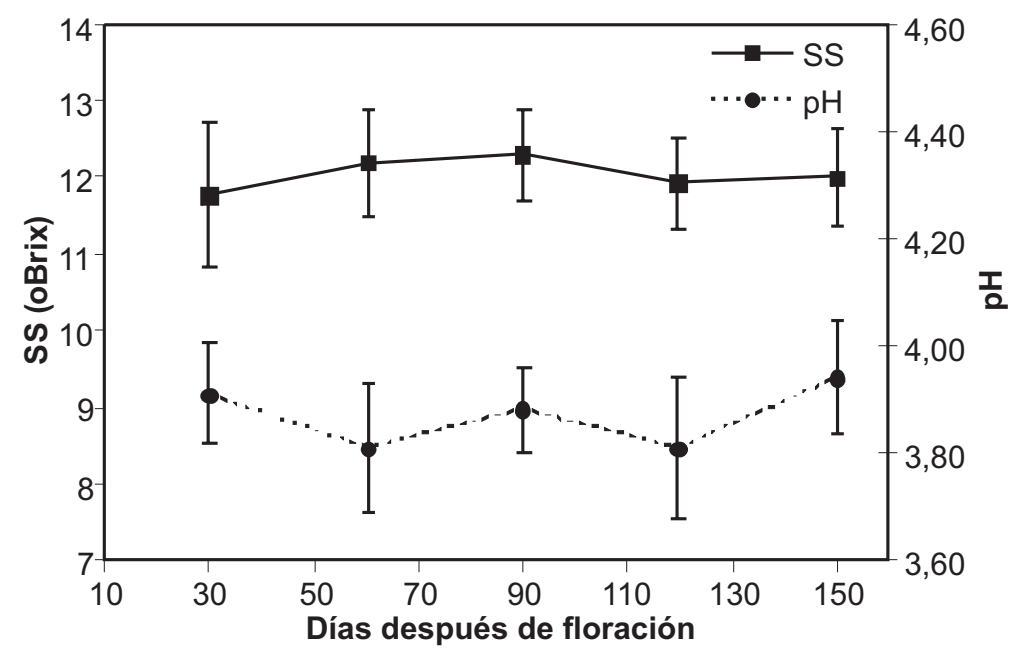

FIGURA 4 - Comportamiento de los sólidos solubles (SS) y del pH durante el crecimiento de la pera variedad Triunfo de Viena. Las barras presentan la media \pm sd.

cual indica que estos índices no se pueden considerar aisladamente para determinar el momento de la cosecha, debido a su poca sensibilidad para el caso de la pera. La determinación de los sólidos solubles debe utilizarse conjuntamente con la acidez titulable para que así tenga utilidad en la definición del momento de la cosecha. Para peras de la variedad Shinsseiki, Lombardi et al. (2000) muestran un comportamiento creciente para los SS, hasta alcanzar $11,3^{\circ}$ Brix en el momento de la cosecha. Seibert et al. (2000) reportan valores de $11,5^{\circ}$ Brix para peras de la variedad Packham's Triumph, los cuales son similares a los encontrados en el presente trabajo $\left(12^{\circ} \mathrm{Brix}\right)$. Otras variedades registran también valores similares en el momento de la cosecha, como es el caso de las variedades Carrick, con $11,7^{\circ}$ Brix (Coutinho et al., 2003), Williams con $11,1^{\circ}$ Brix (Calvo, 2004), Barlett con 12,8Brix (Marsal et al., 2000).

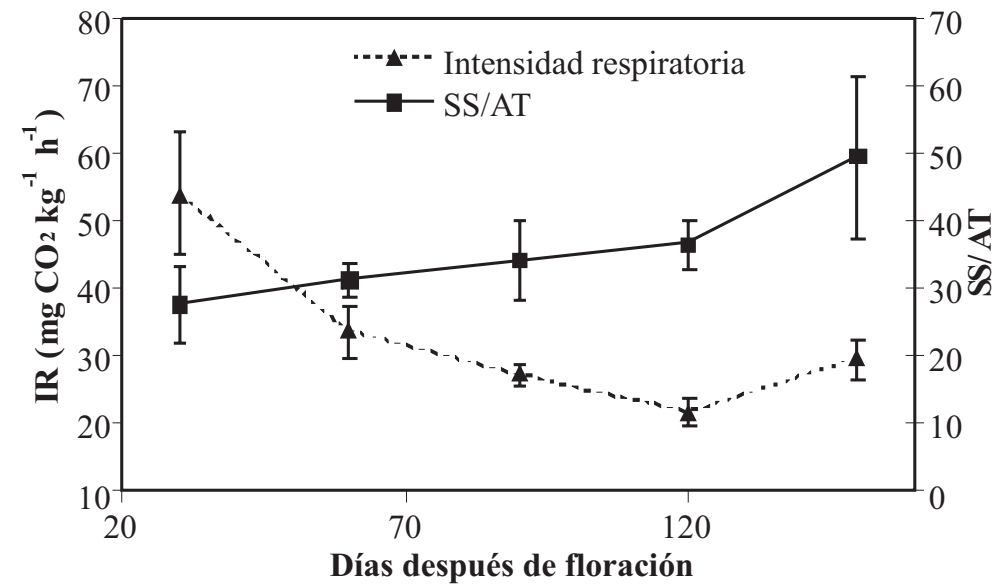

FIGURA 5 - Comportamiento de la intensidad respiratoria (IR) y la relación de madurez (SS/AM) durante el crecimiento de la pera variedad Triunfo de Viena. Las barras presentan la media \pm sd.

La tendencia de la relación de madurez (RM) en el periodo precosecha para frutos de pera aumenta a medida que el fruto crece y se desarrolla, como se observa en la Figura 5, ajustándose al comportamiento de los productos hortofrutícolas, como es reportado por Pantastico (1979).

En la formación y desarrollo inicial del producto la intensidad respiratoria (IR) es elevada y más dispersa entre los diferentes frutos, pero a medida que estos crecen la IR disminuye haciéndose cada vez más homogénea entre los diferentes frutos de la misma edad, al pasar de un CV de 16,9\% en el día 30, a un CV de 8,8\% en el día 150, antes de la cosecha, como se observa en la Figura 5. Con base en la IR registrada a los 120 días (21,70 mg CO $\left.\mathrm{kg}^{-1} \mathrm{~h}^{-1}\right)$ y la IR a los 150 días (29,60 mg CO $\mathrm{kg}^{-1} \mathrm{~h}^{-1}$ ), hacen pensar que el momento adecuado para la cosecha corresponde a una edad intermedia a estas dos, donde posiblemente se encuentre la menor intensidad respiratoria del periodo precosecha. Días de cosecha después de floración similares, son reportados para las variedades Le France (Mwaniki et al., 2005), Barlett (Marsal et al., 2000) y Shinsseiki (Lombardi et al., 2000).

Propiedades físicas de cosecha. La pera variedad Triunfo de Viena, cuyas dimensiones características promedio en el momento de la cosecha son 87,3 mm, 85,8 mm y 80,8 mm, presenta forma esférica, lo cual es ratificado por los valores cercanos a uno, encontrados para esfericidad y redondez (Mohsenin, 1986), siendo estas de 0,886 y 0,899 respectivamente, como se observa en la Tabla 1, determinándose un tamaño medio de la pera de $84,6 \pm 6,5 \mathrm{~mm}$, con CV relativamente uniformes de 7,7\% para el tamaño, 6,9\% para la esfericidad y de 6,2\% para la redondez.

El peso en el momento de la cosecha fue el parámetro físico con mayor dispersión, con un CV de 18,7\%, indicando una baja uniformidad en el tamaño del fruto, en el momento de la cosecha. La densidad aparente (Da) de este producto fue de $540 \mathrm{~kg} \mathrm{~m}^{-3}$, con un

TABLA 1 - Propiedades físicas de la pera variedad Triunfo de Viena, en la cosecha.

\begin{tabular}{|c|c|c|c|c|c|c|c|c|c|}
\hline PARÁMETRO & \multicolumn{3}{|c|}{ MEDIA } & \multicolumn{3}{|c|}{ sd } & \multicolumn{3}{|c|}{ CV (\%) } \\
\hline \multirow{2}{*}{ Dimensiones (mm) } & A & B & $\mathrm{C}$ & A & B & $\mathrm{C}$ & A & B & $\mathrm{C}$ \\
\hline & 87,3 & 85,8 & 80,8 & 7,4 & 6,4 & 5,7 & 8,5 & 7,5 & 7,0 \\
\hline Tamaño* (mm) & & 84,6 & & & 6,5 & & & 7,7 & \\
\hline Peso (g) & & 312,3 & & & 58,4 & & & 18,7 & \\
\hline Esfericidad & & 0,886 & & & 0,061 & & & 6,9 & \\
\hline Redondez & & 0,899 & & & 0,056 & & & 6,2 & \\
\hline Área superficial (cmin) & & 178,07 & & & 7,4 & & & 4,2 & \\
\hline $\mathrm{Da}\left(\mathrm{kg} \mathrm{m}^{-3}\right)$ & & 540 & & & 2,00 & & & 0,37 & \\
\hline $\operatorname{Dr}\left(\mathrm{kg} \mathrm{m}^{-3}\right)$ & & 1040 & & & 14,00 & & & 1,3 & \\
\hline Porosidad (\%) & & 47,54 & & & 0,48 & & & 1,0 & \\
\hline
\end{tabular}

*Tamaño: $(\mathrm{A}+\mathrm{B}+\mathrm{C})$ / 3. Da: Densidad aparente. Dr: Densidad real sd: Desviación estándar.

CV: Coeficiente de Variación 
TABLA 2 - Modelos para la determinación del As, en la pera variedad Triunfo de Viena.

\begin{tabular}{lcc}
\hline MÓDELO & ECUACIÓN & $\mathbf{r}^{2}$ \\
\hline Lineal & $\mathrm{As}=0,4625 \mathrm{~W}+61,6208$ & 0,947 \\
Logarítmico & $\mathrm{As}=111,828 \mathrm{Ln}(\mathrm{W})-436,1$ & 0,916 \\
Cuadrática & $\mathrm{As}=0,0002 \mathrm{~W}^{2}+0,3319 \mathrm{~W}+77,5644$ & 0,948 \\
Potencial & $\mathrm{As}=5,5047 \mathrm{~W}^{0,6301}$ & 0,937 \\
Exponencial & $\mathrm{As}=91,7544 * \mathrm{e}^{0,0026 \mathrm{~W}}$ & 0,942 \\
\hline
\end{tabular}

As: Área superficial $\left(\mathrm{cm}^{2}\right) . \quad$ W: Peso del fruto $(\mathrm{g}) . \quad \mathrm{r}^{2}$ : Coeficiente $\quad$ de determinación

coeficiente de variación bajo, situación similar encontrada para la densidad real (Dr), donde se encontró un CV de 1,3\% para una media de $1040 \mathrm{~kg} \mathrm{~m}^{-3}$. La porosidad del producto encontrada fue de $47,54 \%$, factor de importancia para el almacenamiento del producto, así como para el diseño de empaques.

El área superficial encontrada para la pera variedad Triunfo de Viena, fue de 178,07 cm², parámetro que presentó una relación directa con el peso de la fruta, como se observa en la Tabla 2, donde se relacionan diferentes modelos para determinar el área superficial, con factores de determinación $\left(\mathrm{r}^{2}\right)$ superiores a 0,9 , mostrando un mejor comportamiento para los modelos cuadrático y lineal.

Correlación de parámetros. La Tabla 3 presenta la matriz de coeficientes de correlación (CC) de los índices de cosecha de pera (variedad Triunfo de Viena). De este análisis se determina que la acidez titulable (AT) disminuye a medida que los frutos de pera se desarrollan y crecen en la planta; esta afirmación la sustenta la alta correlación inversa existente entre el peso del producto y su contenido de ácido málico durante el periodo precosecha (CC $=-0,767)$. A medida que el fruto se desarrolla y crece en la planta, la RM se incrementa e indica que el fruto va madurando, como lo muestra el CC de 0,684 demostrando que existe una relación directa entre el crecimiento del fruto y su proceso de maduración. De igual manera entre la RM y la AT, el CC de -0,877 indica que a medida que el fruto madura su acidez titulable disminuye. La pérdida de turgencia de los frutos a medida que estos crecen, permite establecer una relación directa entre la variación de la firmeza y la acidez titulable $(C C=0,729)$ e inversa con el peso $(C C=-0,896)$ es decir, a medida que el fruto crece disminuye su firmeza y su acidez titulable.

La IR de la pera en el periodo precosecha presenta una alta correlación directa con la AT $(C C=0,494)$ y la firmeza $(C C=0,644)$. Por otra parte, la IR presenta una relación inversa con la RM $(C C=-0,432)$ y el peso $(C C=-0,576)$. Estos resultados indican que a medida que el fruto crece y madura en la planta su IR, la firmeza y la AT, disminuyen con el tiempo, hasta el momento de la cosecha.

Los bajos valores de los CC entre los diferentes índices de cosecha y los SS, así como los relacionados con el PH, muestran que no son factores determinantes para establecer el momento adecuado de la cosecha del producto.

TABLA 3 - Coeficientes de correlación de los índices de cosecha para la pera variedad Triunfo de Viena.

\begin{tabular}{|c|c|c|c|c|c|c|c|}
\hline FACTOR & Peso & IR & SS & $\mathbf{A T}$ & SS/AT & Firmeza & pH \\
\hline Peso & 1 & $-0,573 * *$ & $-0,012$ & $-0,767^{* *}$ & $0,684 * *$ & $-0,896^{* *}$ & 0,154 \\
\hline IR & & 1 & $-0,156$ & $0,494 * *$ & $-0,432 * *$ & $0,644 * *$ & 0,127 \\
\hline SS & & & 1 & 0,039 & $0,249 *$ & $-0,011$ & $-0,280^{*}$ \\
\hline $\mathbf{A T}$ & & & & 1 & $-0,877 * *$ & $0,729 * *$ & $-0,313 * *$ \\
\hline SS/AT & & & & & 1 & $-0,660 * *$ & $0,340 * *$ \\
\hline Firmeza & & & & & & 1 & $-0,012$ \\
\hline pH & & & & & & & 1 \\
\hline
\end{tabular}

IR: Intensidad respiratoria. SS: Sólidos solubles. AT: Acidez titulable.

* La correlación es significativa a un nivel de 0,05.

** La correlación es significativa a un nivel de 0,01.

\section{CONCLUSIONES}

1. En la formación y desarrollo inicial del producto la intensidad respiratoria es elevada y más dispersa, pero a medida que las peras crecen la intensidad respiratoria disminuye haciéndose cada vez más homogénea entre los diferentes frutos de la misma edad, lo cual se ajusta al comportamiento de los frutos en general.

2. La pera variedad Triunfo de Viena presenta forma esférica y su área superficial es función directa del peso de la fruta.

3. El peso (312,3 $\pm 58,4 \mathrm{~g})$, la firmeza $(67,82 \pm 6,88 \mathrm{~N})$, la acidez titulable $(0,24 \pm 0,05 \%$ de ácido málico) y la relación SS/AT (49,36 \pm 11,98), son parámetros adecuados para establecer el momento adecuado para la recolección de la pera variedad Triunfo de Viena.

4. En general para el periodo precosecha, los SS y el pH presentan una tendencia aproximadamente constante y de baja dispersión, lo cual indica que estos índices no deben considerarse aisladamente para determinar el momento de la cosecha, debido a la poca sensibilidad que presentan.

5. La combinación de diferentes parámetros físicos y fisiológicos, permiten establecer de manera confiable el momento apropiado para la cosecha de la pera, así como las características básicas para el diseño de empaques e instalaciones de almacenamiento.

\section{REFERENCIAS}

CALVO, G. Efecto del 1-metilciclopropeno (1-MCP) en pera variedad Williams cosechadas con dos estados de madurez. Revista de
Investigaciones Agropecuarias, Buenos Aires, v.33, n.2, p.3-26, 2004.

COUTINHO, E.F.; MALGARIM, M.B.; SOUZA, E.L. de; TREPTOE, R.O. Qualidade pós-colheita da pêra (Pyrus communis L.) cultivar Carrick, submetida a diferentes condições de armazenamento. Revista Brasileira de Fruticultura, v.25, n.3, p.417-420, 2003.

FOOD AND AGRICULTURE ORGANIZATION OF THE UNITED NATIONS - FAO. Base de datos estadísticos - Faostat Agriculture. 2005

GARRIZ, P.I.; COLAVITA, G.M.; ALVAREZ, H.L. Fruit and spur leaf growth and quality as influenced by low irradiance levels in pear. Scientia Horticulturae, v.77, p.195-205. 1998.

KABAS, O.; OZMERZI, A. Comparing of the bruise volume calculation methods for Ankara pear (Pyrus communis L.). Journal of Food Engineering, In press. 2005

KAPPEL, F.; NEILSEN, G.H. Relationship between light microclimate, fruit growth, fruit quality, specific leaf weight and $\mathrm{N}$ and $\mathrm{P}$ content of spur leaves of 'Bartlett' and 'Anjou' pear. Scientia Horticulturae, v.59, p.187-196, 1994

KAPPEL, F.; FISHER, F.R.; HOGUE, E.J. Ideal pear sensory attributes and fruit characteristics. HortScience, v.30, n.5, p.988-993, 1995.

LOMBARDI, S.R.B.; MORAES, D.M. de; CAMELATTO, D. Avaliação do crescimento e da maturação pós-colheita de pêras da cultivar Shinsseiki. Pesquisa Agropecuária Brasileira, v.35, n.12, p.23992405, 2000.

MARSAL, J.; RAPOPORT, H.F.; MANRIQUE, T.; GIRONA, J. Pear fruit growth under regulated deficit irrigation in container-grown trees. 
Scientia Horticulturae, v. 85, p.243-259, 2000.

MOHSENIN, N.N. Physical properties of plant and animal material. v.1. New York: Gordon and Breach Science Publisher. 1986. 734 p.

MWANIKI, M.C.; MATHOOKO, F.M.; MATSUZAKI, M.; HIWASA, K.; TATEISHI, A.; USHIJIMA, K.; NAKANO, R.; INABA, A.; KUBO, Y. Expression characteristics of seven members of the $\beta$ galactosidase gene family in La France pear (Pyrus communis L.) fruit during growth and their regulation by 1-methycyclopropene during postharvest ripening. Postharvest Biology and Technology, v.36, p.253-263, 2005.

PANTASTICO, E.B. Fisiología de la postrecolección, manejo y utilización de frutas y hortalizas tropicales y subtropicales. México D.F.: Editorial CECSA . 1.979.
PARK, K.J.; BIN, A.; BROD, F.P.B.; PARK, T.H. Osmotic dehydratation kinetics of pera D'anjou (Pyrus communis L.). Journal of Food Engineering, v.52, p.293-298, 2002.

PARRA C., A.; HERNÁNDEZ H., J.E. Fisiología Postcosecha de frutas y hortalizas. Bogotá. Universidad Nacional de Colombia. Facultad de Ingeniería. 1997. 64p.

SEIBERT, E.; BARRADAS, C.I.N.; ARAÚJO, P.J, de; BENDER, R.N. Efeito de ethehon e da frigoconservação na maturação de pêras cv. Packham's Triumph. Pesquisa Agropecuária Brasileira, v.35, n.1, p.55-62, 2000. 